\title{
Performance Evaluation Method Proposal for High Performance ICT Systems and Networks
}

\author{
${ }^{1}$ Jozef B. Lewoc, ${ }^{2}$ Antoni Izworski, ${ }^{3}$ Slawomir Skowronski, ${ }^{4}$ Antonina Kieleczawa, \\ ${ }^{5}$ Peter Kopacek, ${ }^{6}$ Nicolae Bulz \\ ${ }^{I}$ Design, Research and Translation Agency Leader Wroclaw, Poland \\ ${ }^{2}$ Institute of Automation and Robotics Wroclaw University of Technology Wroclaw, Poland \\ ${ }^{3}$ Informatics and Management Faculty Wroclaw University of Technology Wroclaw, Poland \\ ${ }^{4}$ Institute for Power System Automation (IASE) Wroclaw, Poland \\ ${ }^{5}$ Institute for Handling Devices and Robotics Vienna University of TechnologyVienna, Austria \\ ${ }^{6}$ Victoria University Centre for Strategic Economic Studies, Melbourne, Victoria, Australia National Defence College Bucharest, \\ Romania World Economy Institute/ (NERI)/ Romanian Academy Bucarest, Romania Interdisciplinary Research Group / \\ Romanian Academy Bucharest Romania
}

\begin{abstract}
This paper presents a predicted functional architecture of an emerging application of High Performance Computing and Communication (HPCC) application: the integrated manufacturing and management system. The computer network was defined for a case study of big manufacturer of household appliances. To evaluate and/or predict performance of this network, a method developed earlier for actual computer networks, i.e. packet switching networks with end-to-end acknowledgement was applied. The network is modelled as a set of closed routes consisting of a user and a series of service stations (communication links, switches, host processes). The paper describes the investigations carried out for the case study. Some consideration is given to the performance evaluation accuracy, basing on the validation work results obtained from simulation and measurements on the Polish pilot wide area network. The method is proposed for performance evaluation/prediction for high performance computing and communication systems/networks, as an alternative to widely advertised but failing in actual practice closed queuing network methods.
\end{abstract}

\section{INTRODUCTION}

The Computer-Integrated Manufacturing and Management (CIMM) systems are severely needed by prospective users, especially big and medium manufacturing enterprises. Many people start to realize that development and implementation of such systems is necessary for the manufacturing enterprises to be duly monitored and run in some optimum way, in accordance with some objective operational criteria. In opinion of the executive staff of many manufacturing enterprises visited by the authors, such optimization of complete enterprises is or will be soon a prerequisite for their existence on the highly competitive market.

In spite of that, big manufacturers and vendors of the Computer Integrated Management (CIM) systems, having at their disposal financial resources sufficient to develop and implement at least pilot CIMM systems, are no apt to enter the manufacturing domain. The basic reason for that seems to be the fact that the big CIM system manufacturers and vendors do not possess at their disposal the designers and implementers educated and experienced enough to cope with both manufacturing and management issues. Such persons are, however, available; paradoxically, many of them are available in poorly developed countries where the designers must have possess a wide scope of experience in order that they are successful; unfortunately, they are dispersed in various industrial and/or research organisations and they do not have at their disposal the financial resources needed to develop novel high performance ICT systems of the CIMM type.

In such circumstances, a reasonable duty of research and development workers seems to be to carry out the initial work for the prospective project of CIMM systems, even if no financing is available for such work. This work may be considered as a volunteer work or a work for the society (as it is called in the case-study country of Poland) that have financed their earlier research and development work or as an investment in their own future: the designers involved early in the prospective CIMM projects will have high chances to be included in the project execution team if and when the pilot CIMM project is established.

This initial work should also include the definition of the system topology (here, the wireless solution seems to be the most appropriate one for the actual solution since the systems of the current state of art should be applied for the novel solutions) and designer-friendly investigation methods to ensure adequate performance for hardware and software solutions under design. 


\section{Performance Evaluation Method Proposal for High Performance ICT Systems and Networks}

A team of ICT experts and process technologists was established and worked out a reasonable, safe approach to development of CIMM systems, called "by-thinking", based on the possibly fast process of education of the design and development Team in the domain of manufacturing and management, development of the first thread through CIMM and working out the concept, procedures and standards for future CIMM systems basing on that knowledge and experience [1-7]. This approach creates the basis for this paper.

It should be mentioned here, that there is in progress another approach to design and development of the emerging of high performance computing and communication applications being CIMM systems. This method is based on high investments at the beginning, development of the CIMM idea and development standards at the very beginning of the design process, on separation of the manufacturing and management concerns and on using separate systems for solving the managerial and manufacturing problems. The approach is called "by-organizing". The authors do not think that it is appropriate to criticize people using other approach in their work so they are not cited in the present paper.

\section{EXEMPLARY CIMM ARCHITECTURE}

The pilot CIMM system was designed initially several years ago for the ex-biggest Polish manufacturer of household appliances, Polar, Wroclaw (the Enterprise), employing several thousand people. The target organisational structure of the Enterprise would be that depicted in Fig. 1.

For the general Enterprise organisation presented in Fig. 1, the wireless hardware network architecture of the CIMM system, depicted in Fig. 2, has been proposed. Logically, the architecture is based on the wireless metropolitan network operating under the TCP/IP protocol suite [8] and on the process control and monitoring network of the Echelon Control Networks type [9].

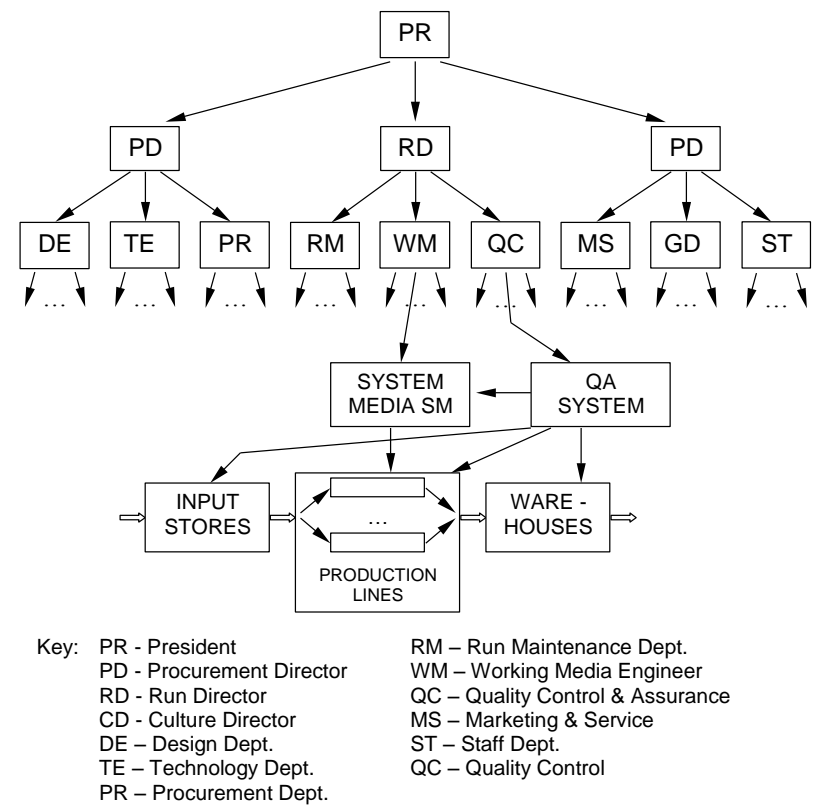

Fig. 1. General organisational diagram of the enterprise

\section{THE NETWORK UNDER INVESTIGATION}

It is assumed that the time needed for transfer of data via any switch (server, gateway, node or router) is insignificant in comparison with the time needed to transfer the data via a co-operating data link. This is in conformity with the specifications of actual hardware proposed for the CIMM system since data is transferred via network switches as 8-bit byte blocks transferred via fast direct memory access (DMA) channels and internal processing of the data is usually connected only with the message headers.

However, the method proposed enables to consider the delays in wireless network switches though the calculations may be somewhat more complicated. 


\section{Performance Evaluation Method Proposal for High Performance ICT Systems and Networks}

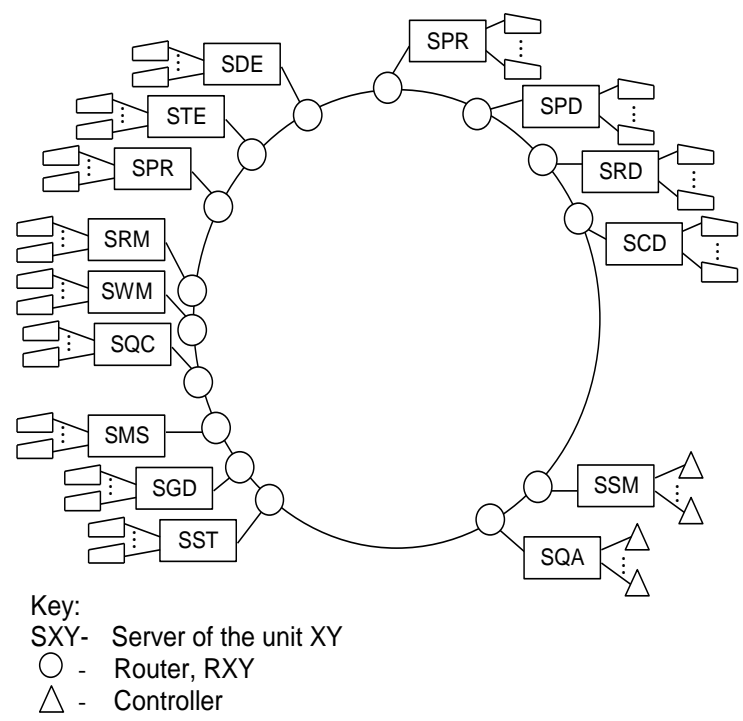

Fig. 2. Hardware architecture of the CIMM system

The hardware structure of the wireless CIMM system has been presented in Fig. 2. The case-study network depicted in Fig. 2 is composed of 30 switches (15 routers and 15 servers). Let the individual switches and data links be numbered as shown in Fig. 3 (server Nos: 1- 15, router Nos: 16-30, link pairs (for both transmission directions): $(1,2)-(85,86)$.

Upon the wireless network of Fig. 3, there is stretched a set of closed routes, i.e. the calls from a user to its server and in the opposite direction. . Exemplary closed routes in the CIMM network are the connections between a Marketing \& Service Department worker and the general enterprise database (the President's database) (closed route No. $s=166$; ref. Fig. 4a) and between a technologist and the system Media database (closed route No. s = 279; ref. Fig. 4b).

Note: it is assumed that any p-th server, $\mathrm{p} \in 1, \ldots, 15$ (ref. Fig. 3 ), is able to support 20 closed routes of numbers $\mathrm{s}=(\mathrm{p}-1) 20,(\mathrm{p}-1) 20+1, \ldots,(\mathrm{p}-1) 20+19$.

Note also that the network described here as a set of closed routes may be conveniently considered as a case of the Kelly networks [6] where the entity (packet) sojourn time in any switch is a function of the number of packets in all routes in the network. However, the classic queuing theory does not look for solutions for the Kelly networks $[7,8]$. 


\section{Performance Evaluation Method Proposal for High Performance ICT Systems and Networks}

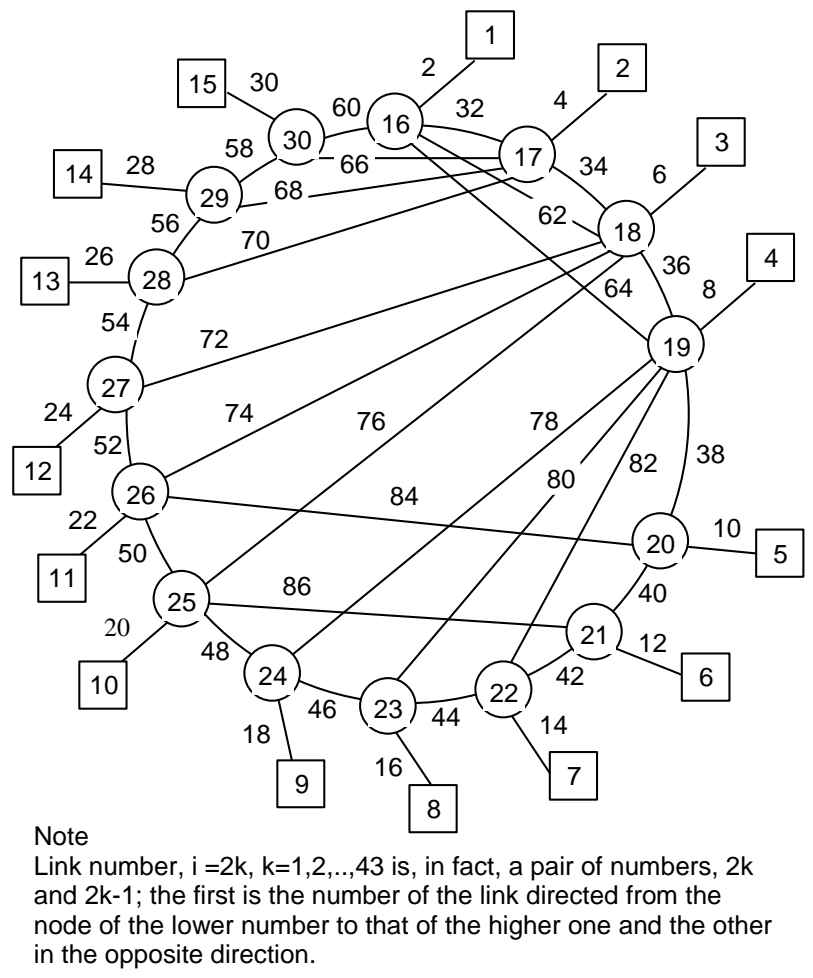

Fig. 3. Numbering of switches and links

Therefore, it was decided to adapt the approximate performance evaluation method [6], developed and validated earlier for telephone computer and communication networks with end-to-end acknowledgement, to the cases of the CIMM system implemented on wireless networks and to apply the method for performance evaluation for the case study under discussion.

\section{THE APPROXIMATIONS}

The reference [6] is hardly available now. Therefore, some basic assumptions and results will be repeated in the present paper.

In addition to the denotations defined in Fig. 3, the following will be used in the present paper:

$A_{i}$ is the set of closed routes beginning at the $i$-th link, such that the s(i)-th user is connected directly to the $i$-th link:

$$
A_{i}=\left\{s_{i, 1}, s_{i, 2}, \ldots, s_{i, a_{i}}\right\}
$$

$B_{i}$ is the set of closed routes passing via the $i$-th link but not beginning at that link:

$$
B_{i}=\left\{z_{i, 1}, z_{i, 2}, \ldots, z_{i, b_{i}}\right\}
$$




\section{Performance Evaluation Method Proposal for High Performance ICT Systems and Networks}

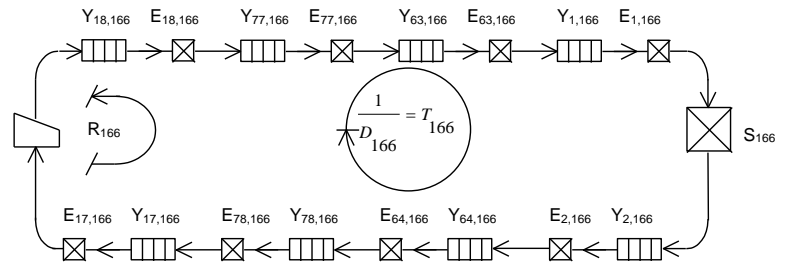

a) Exemplary closed route of a Marketing \& Service employee using general enterprise database

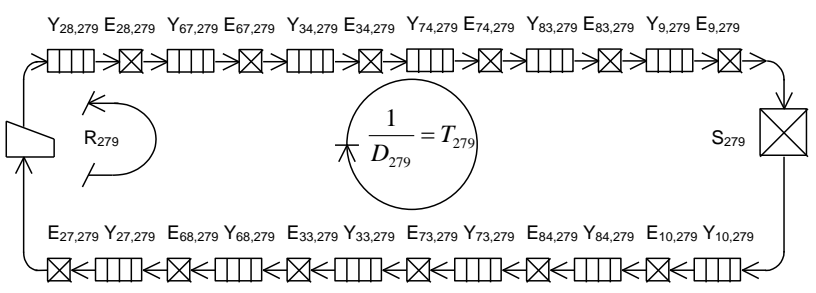

b) Exemplary closed route of a technologist using the System Media

Key:

$\mathrm{s} \in \mathrm{S}=1, \ldots, 299 ; \mathrm{i} \in \mathrm{I}=1, \ldots, 86$

$\mathrm{R}_{\mathrm{s}}=$ round route (trip) delay for $\mathrm{s}-$ th closed route

$\mathrm{T}_{\mathrm{s}}=\mathrm{s}$-th closed route cycle time

$\mathrm{D}_{\mathrm{s}}=\mathrm{s}$-th closed route throughput

$\mathrm{Y}_{\mathrm{i}, \mathrm{s}}=$ waiting time of $\mathrm{s}$-th closed route to $\mathrm{i}$-th link

$\mathrm{E}_{\mathrm{i}, \mathrm{s}}=$ service time of $\mathrm{s}$-th closed route to $\mathrm{i}$-th link

$\mathrm{M}_{\mathrm{s}}=\mathrm{s}$-th closed route thinking time

$\mathrm{S}_{\mathrm{s}}=\mathrm{s}$-th closed loop ultimate service time

Note: Upper case letters denote random variables while lower case letters - corresponding mean values

Fig. 4. Exemplary closed routes

The link preceding the $i$-th link in the $s$-th closed route is denoted by $h=h_{s}(i)$, provided that $s \in B_{i}$.

The set of closed routes parallel to the s-th closed route in the i-th link is defined by (3).

$$
C_{i}=B_{i} \cap\left(A_{h} \cup B_{h}\right)
$$

The power of $\mathrm{C}_{\mathrm{i}}$ is given by (4).

$$
\overline{C_{i}}=c_{i}
$$

The mean cycle time of closed routes encountered by the s-th route at the i-th link is approximated by (5).

$$
\bar{t}_{i, s}=\frac{a_{i}+b_{i}-1}{\sum_{l \in\left\{A_{i} \cup B_{i}\right\}\langle\{s\}} \frac{1}{t_{i}}} ; \frac{0}{0}=0 ;
$$




\section{Performance Evaluation Method Proposal for High Performance ICT Systems and Networks}

The mean waiting time of the closed routes encountered by the s-th route at the i-th link is given by (6).

$$
\bar{y}_{i, s}=\frac{\bar{t}_{i, s}}{\left(a_{i}+b_{i}-1\right)}\left(\sum_{l \in\left\{A_{i} \cup B_{i}\right\} \backslash\{s\}} \frac{y_{i, l}}{t_{i}}\right) ;
$$

The mean service time for the closed routes encountered by the s-th route at the i-th link is given by (7).

$$
\bar{e}_{i, s}=\frac{\bar{t}_{i, s}}{\left(a_{i}+b_{i}-1\right)}\left(\sum_{l \in\left\{A_{i} \cup B_{i}\right\} \backslash\{s\}} \frac{e_{i, l}}{t_{l}}\right) ;
$$

The mean thinking time for the closed routes encountered by the s-th route at the i-th link is given by (8).

$$
\bar{m}_{i, s}=\frac{\bar{t}_{i, s}}{\left(a_{i}+b_{i}-1\right)}\left(\sum_{\left.l \in\left\{A_{i} \cup B_{i}\right\} \backslash s\right\}} \frac{m_{i, l}}{t_{l}}\right) \text {; }
$$

The definitions of the mean values defined above for the set of closed routes that are not parallel to the s-th route are as follows:

$$
\begin{gathered}
\bar{t}_{i, s}^{\prime}=\frac{a_{i}+b_{i}-c_{i}}{\sum_{l \in\left\{A_{i} \cup B_{i}\right\} \backslash C_{i}} \frac{1}{t_{i}}} ; \\
\bar{y}_{i, s}^{\prime}=\frac{\bar{t}_{i, s}^{\prime}}{a_{i}+b_{i}-c_{i}} \sum_{l \in\left\{A_{i} \cup B_{i}\right\} \backslash C_{i}} \frac{y_{i, l}}{t_{i}} ; \\
\bar{e}_{i, s}^{\prime}=\frac{\bar{t}_{i, s}^{\prime}}{a_{i}+b_{i}-c_{i}} \sum_{l \in\left\{A_{i} \cup B_{i}\right\} \backslash C_{i}} \frac{e_{i, l}}{t_{l}} ; \\
\bar{m}_{i, s}^{\prime}=\frac{\bar{t}_{i, s}^{\prime}}{a_{i}+b_{i}-c_{i}} \sum_{l \in\left\{A_{i} \cup B_{i}\right\} \backslash C_{i}} \frac{m_{i, l}}{t_{l}} ;
\end{gathered}
$$

For all closed routes, the balance equations (13) have been defined.

The set of equations (5) - (13) enables to compute iteratively the basic unknown mean values of the network performance, i.e. the closed route cycle time, $\mathrm{t}_{\mathrm{s}}$, the mean throughput in the s-th closed route, $d_{s}=\frac{1}{t_{s}}$, and the round-trip delay, $r_{s}=t_{s}-m_{s}$.

$\bigcap_{s \in S}\left(t_{s}=\sum_{i \in I_{s}}\left(y_{i, s}+e_{i, s}\right)\right)$ 


\section{Performance Evaluation Method Proposal for High Performance ICT Systems and Networks}

where $\mathrm{S}$ is the set of all closed loops, $\bar{S}=v$;

$$
\begin{aligned}
& \bigcap_{s \in A_{i}}\left(y_{i, s}=\frac{\left(a_{i}+b_{i}-1\right) \bar{e}_{i, s}^{2}}{\bar{t}_{i, s}\left(1-\frac{e_{i, s}}{t_{s}}\right)}\left(\frac{1}{2}+\right.\right. \\
& +\left(a_{i}+b_{i}-2\right)\left(1-\frac{\bar{m}_{i, s}}{\bar{t}_{i, s}-\bar{e}_{i, s}}\right) \frac{\bar{y}_{i, s}}{\bar{t}_{s}-\bar{e}_{i, s}}+ \\
& \left.+\left(a_{i}+b_{i}-1\right)\left(1-\frac{\bar{m}_{i, s}}{\bar{t}_{i, s}-\overline{\bar{e}}_{i, s}}\right) \frac{\frac{\bar{e}_{i, s}}{2}}{\bar{t}_{i, s}-\bar{e}_{i, s}}\right) ;(13) \\
& \bigcap_{s \in B_{i}}\left(y_{i, s}=\frac{\left(a_{i}+b_{i}-c_{i}\right) \bar{e}_{i, s}^{\prime 2}}{\bar{t}_{i, s}^{\prime}\left(1-\frac{e_{i, s}}{t_{s}}\right)}\left(\frac{1}{2}+\left(a_{i}+b_{i}-c_{i}-1\right)\right.\right. \\
& \left(1-\frac{\bar{m}_{i, s}^{\prime}}{\bar{t}_{i, s}^{\prime}-\bar{e}_{i, s}^{\prime}}\right) \frac{\bar{y}_{i, s}^{\prime}}{\bar{t}_{i, s}^{\prime}-\bar{e}_{i, s}^{\prime}}+ \\
& \left.+\left(a_{i}+b_{i}-c_{i}\right)\left(1-\frac{\bar{m}_{i, s}^{\prime}}{\bar{t}_{i, s}^{\prime}-\bar{e}_{i, s}^{\prime}}\right) \frac{\frac{\bar{e}_{i, s}^{\prime}}{2}}{\bar{t}_{i, s}^{\prime}-\bar{e}_{i, s}^{\prime}}\right) \text {; }
\end{aligned}
$$

\section{VALIDATION OF THE APPROXIMATIONS}

\section{A. Validation with accurate results for cyclic queuing systems}

The problem of performance evaluation of homogeneous star-topology networks is the same as that investigated for wireless queuing networks. The accurate solutions in the form of limit probabilities of the entity (packet, request) numbers have been known since early seventies. These results, for exponential thinking time, were employed to validate the approximations presented above.

Several hundred comparisons were done for the number of the customers (closed routes), v, changing between 2 and several dozen. The relative error of the mean cycle time, $t_{s}$, calculated for the set of comparison reached its maximum (less than 0.03 ) at $\mathrm{v} \approx 14$ and decreased for $\mathrm{v}$ tending to zero or to infinity.

\section{B. Validation with simulation}

In order that the approximations may be validated, a fast Wide-Area-Network simulator was developed on the basis of earlier simulation studies for real-time computer control systems. This simulator was used, primarily, for the Polish pilot wide area network MSK. However, both actual and planned network configurations (including high performance ICT systems and networks that were to operate at much higher transmission volumes and rates) were investigated. In addition, some available foreign simulation results for local and wide area networks were also used for validation.

The number of validation experiments was higher that 500, with the number of closed loops and links equal up to 500 and 100, respectively. The maximum relative error found was always lower than $3.5 \%$.

\section{Validation with measurements}

To investigate MSK and the approximations presented above, an internal communication network measuring tool Sitwa was developed and implemented. It was used, primarily, to validate the simulation results on the existing 


\section{Performance Evaluation Method Proposal for High Performance ICT Systems and Networks}

possible configurations of MSK. The investigations showed that the simplifying assumptions in simulation (e.g. omission of the flow control packets and/or frames) did not result in significant simulation errors. The maximum relative error of the approximations under discussion did not exceed 0.05 .

\section{INTENSIVE TRAFFIC CIMM INVESTIGATION SCENARIO}

To produce some severe traffic conditions for the wireless CIMM system under investigation, it has been assumed that a major failure occurs in the Enterprise, of impact upon all departments. All users operate at one time trying to locate the problem within their responsibility ranges. Most of them (ca. nine per ten) use then the general Enterprise database (SPR), some try to analyse the current process conditions and to communicate with the System Media (SSM) or the Quality Control System (SQA). The basic routing arrays in the closed routes are set up so that the minimum possible number of lines is used in each closed route, provided that data transfer routes follow the hierarchy levels in the Enterprise. The use of the back-up routes may, possibly, produce even more severe traffic conditions. However, they are used only for very severe wireless CIMM system failures. In such cases, however, the main objective for the CIMM system operation is to recover the normal operating conditions as fast as possible and any optimisation of the transmission conditions is not of major interest then.

It is assumed that the data transfer rate in each link is equal to $1 \mathrm{Mbit} / \mathrm{s}$. Each subscriber sends a short message of the mean length 100 bits (bit stuffing, i.e. insertions o a 0 bits after each five 1 bits in a sequence, included) that needs $10^{-4} \mathrm{~s}$ for transmission across any line along the route to the final server, and receives the 100,000 bit-long reply that needs $0.1 \mathrm{~s}$ for the transfer across any link on the route back to the subscriber.

The thinking time mean value, $\mathrm{m}_{\mathrm{s}}$, has been changed from $30 \mathrm{~s}$ down to ca. $0 \mathrm{~s}$; this reflects the changeover from the interrogating transfer mode to the automatic file transfer mode (bulk transfer mode). It will be assumed that the service time at the final station of each closed loop is $0 \mathrm{~s}$. This solution is implied by the fact that all the investigations described referred to the communication network; for possible investigations of the overall computer networks, adoption of relevant distributions/characteristics of service times is possible.

Let $I_{s}=\left\{i_{i, s}, i_{2, s}, \ldots, i_{k_{s}, s}\right\}$ be the set of links passed by the s-th closed route.

\section{EXEMPLARY RESULTS}

The set of equations (5) $\div$ (13) was solved iteratively. The basic user characteristics, $r_{s}$ and $d_{s}$, for the exemplary closed routes, $\mathrm{s}=166$ and $\mathrm{s}=279$, are presented in Figs. $5 \div 8$.

The exemplary round-trip delay $\left(\mathrm{r}_{166}\right)$ curve versus the mean thinking time $\left(\mathrm{m}_{166}\right)$ is shown in Fig. 5.

The $d_{166}$ values (the mean throughput values depicted in Fig. 6) show a definite saturation (congestion) at $\mathrm{m}_{\mathrm{s}}$ values below ca. $0.5 \mathrm{~s}$. If the mean offered load for the 166-th closed route is defined by (14):

$$
t_{166}^{o f f}=\sum_{i \in 166}\left(e_{i, s}+m_{s}\right)
$$

then the increased offered load results in the decreased mean throughput, $\mathrm{d}_{166}$, for the given scenario and for $\mathrm{m}_{\mathrm{s}}<0.5 \mathrm{~s}, \mathrm{~s}=1, \ldots, 300$.

The closed route, $s=279$ (Fig. 7), shows only the acceptable $r_{s}$ values (below $1 \mathrm{~s}$ while the values not greater than $2 \mathrm{~s}$ are acceptable as not annoying ones) and the $\mathrm{d}_{\mathrm{s}}$ value is decreasing monotonically with the offered load increasing $\left(\mathrm{m}_{\mathrm{s}} \rightarrow 0, \mathrm{~s}=1, \ldots, 300\right)$ (Fig. 8).

The reason is that the 279-th closed route does not pass via any link under heavy traffic. Therefore, even when the mean thinking time is decreased towards zero, no saturation (congestion) occurs in the links passed by the 279-th closed route.

The closed route, $s=166$, passes via the most severely loaded links, $i=1$ and $i=2$. This results in that $r_{166}$ reaches more than $4 \mathrm{~s}$ at $\mathrm{m}_{\mathrm{s}}$ values close to 0 (ref. Fig. 5).

If it is assumed that an annoying value (i.e. the value that can not be accepted by the Enterprise employees) of $r_{s}$ is that higher than $2 \mathrm{~s}$, then the $\mathrm{m}_{\mathrm{s}}$ values below some $0.5 \mathrm{~s}$ should be avoided. 
Performance Evaluation Method Proposal for High Performance ICT Systems and Networks

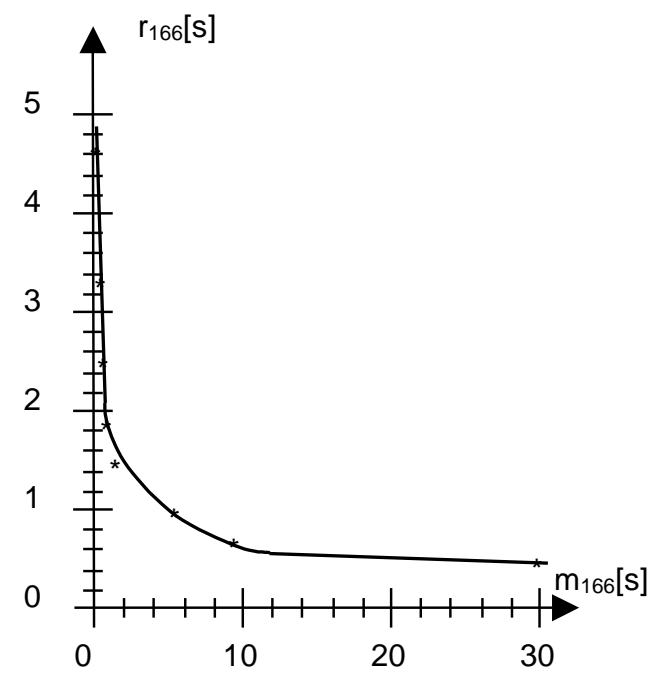

Fig. 5. $r_{166}$ versus $m_{166}$

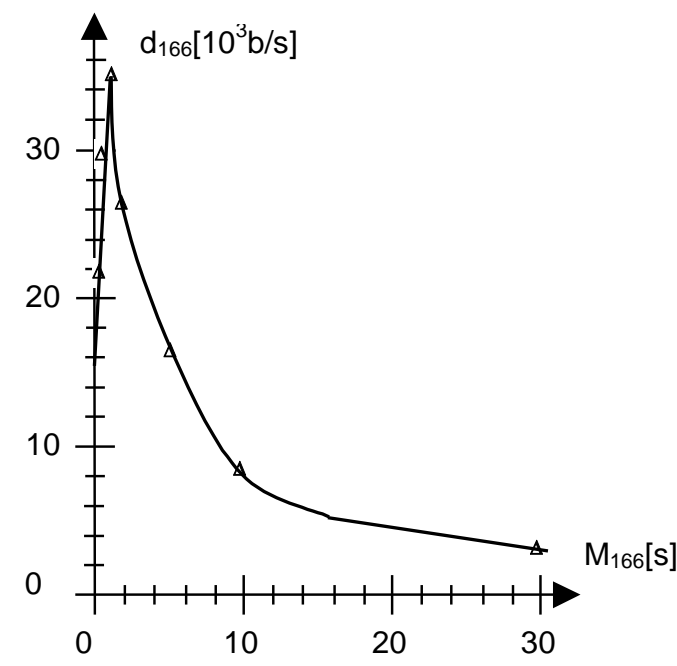

Fig. 6. $\mathrm{d}_{166}$ versus $\mathrm{m}_{166}$ 
Performance Evaluation Method Proposal for High Performance ICT Systems and Networks

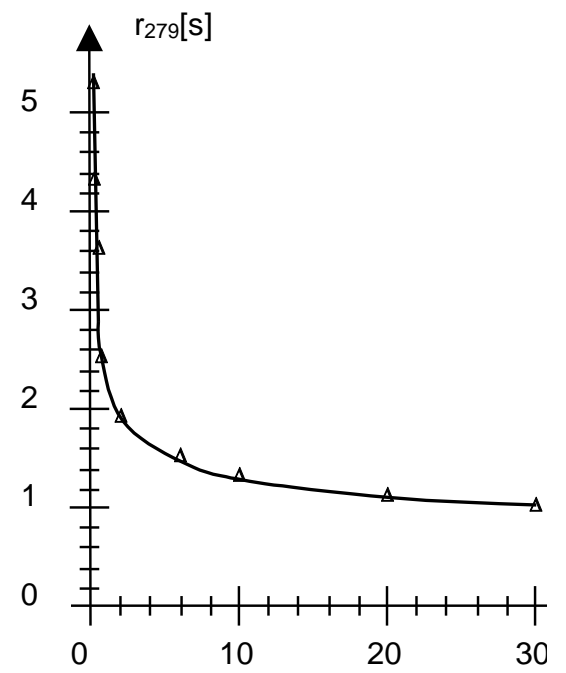

Fig. 7. $\mathrm{r}_{279}$ versus $\mathrm{m}_{279}$

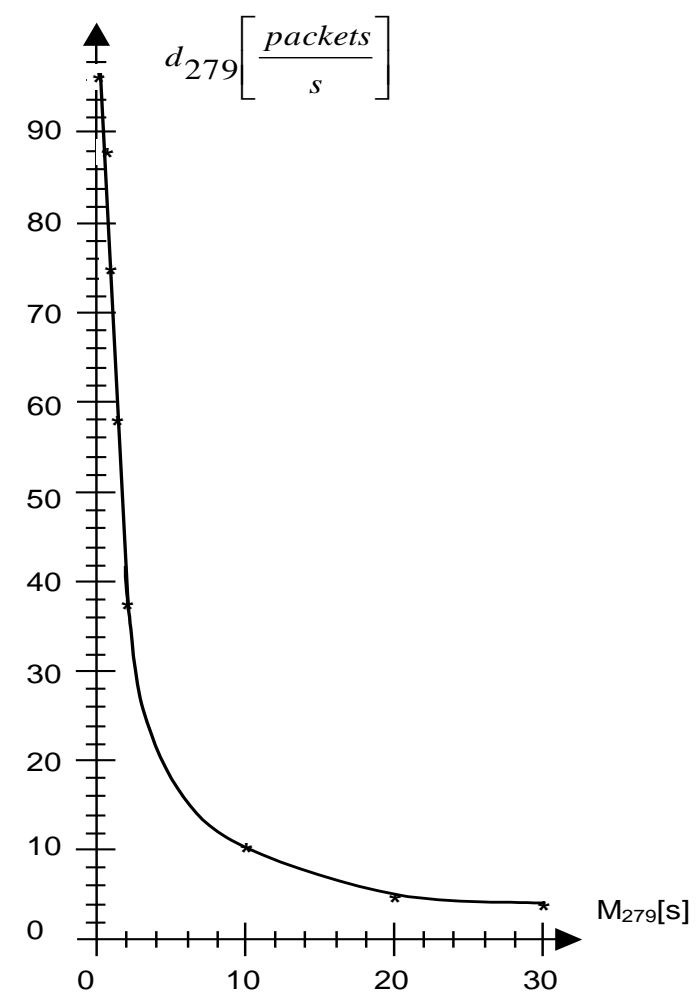

Fig. $8 . d_{279}$ versus $m_{279}$ 


\section{Performance Evaluation Method Proposal for High Performance ICT Systems and Networks}

\section{CONCLUSIONS}

The authors do not claim that the method devised and verified initially some dozen years ago needs no further work. On the contrary, they do realise that the method validated primarily on the pilot Polish Interuniversity Computer Network MSK, operating at medium transmission rates and in a limited configuration, needs further validation and possible tuning. In addition, the method may and should be upgraded: thanks to the rapid growth of the computing power that has happened during the last decade, more accurate approximations may be done for the balance equations (e.g., the simplifying assumption that an entity (packets) may find only one entity of one closed loop in the queue to some link) may be discarded. Then the balance equations become more complicated but may be still solved by the iterative method. And the networks with sliding windows or credit-based flow control may be modelled directly, instead of modelling several loops for credits bigger than 1, as it has been done earlier. In other word, this method should be treated as a proposal of an approach to performance evaluation / prediction of actual of actual ICT systems and networks. The persons truly interested in performance of the relevant ICT system / network may and should adopt their own approximations and develop their own specific solutions.

It is very, very worthwhile. For actual ICT systems and networks, the authors heard many advices, how to investigate; however, they did not see any actual solution. A good example here are the contacts with a conference on computer networks, of the upmost world prestige. The referee's comments, year by year, were: apply MVA, apply MVA, the only method are stochastic Markovian networks, network performance evaluation is infeasible. A reflexion: it is a shame that referees such incompetent in actual ICT systems and networks do happen in prestigious network conference committees.

And a feature of the approach proposed has got a feature particularly precious for investigation of HPCC applications. They belong, for sure, to emerging ICT applications. The performance investigations described in the paper began in late sixties, during design and development of the first Polish application of ICT in process control and coordination for a steel-rod mill, of a complexity and difficultness level not lower than those of present day computer control systems, developed by multi-designer teams. The system was designed by two designers having at their disposal a computer obsolete of some 5-10 years with respect to those available in the West. Thus performance issues were vital. The queuing theory was of a terminological only and not a substantial help. So an event-driven simulator was written and used. It seemed unbelievable for the designers how much they could learn about their own hardware and software solutions just from the development of the model itself. The fashionable analytical methods map the queuing systems (as a matter of fact, which can hardly be considered as isomorphic mappings of actual computer systems/networks) into some hardly understandable domains/spaces thus eliminating this very important educational feature.

\section{REFERENCES}

[1] L. Franasik et al., System Media - a Step toward Computer Integrated Manufacturing and Management Systems, Measurement and Control (AMSE Press), 22 (3), 2001, 37-46.

[2] A. Izworski, J.B. Lewoc and S. Skowronski, Development of Computer Integrated Manufacturing and Management Systems. A. Dolgui, G. Morel, C. Pereira (Eds.), $12^{\text {th }}$ IFAC Symposium on Information Control Problems in Manufacturing, INCOM 2006, Saint Etienne, Elsevier (Oxford, UK), 2007 , p.6.

[3] J.B. Lewoc, A. Izworski and S. Skowronski, ALSIS case study: computer integrated manufacturing and management systems. K. Leviska (Ed.), $1^{\text {st }}$ IFAC Workshop on Applications of Large Scale Systems, Application of Large Scale Industrial Systems (IFAC), ALSIS '06, Helsinki-Stockholm, Elsevier (Oxford, UK), p.6.

[4] J. B. Lewoc, A. Izworski, S. Skowronski, A. Kieleczawa, G. M. Dimirovski, Computer Integrated Manufacturing and Management Systems - An Emerging Application of Control and Management. A. Ristecki (Ed.), IX Conference, ETAI, Ohrid (Macedonia), ETAI Press, 2009 , p. 7.

[5] J. B. Lewoc, A. Izworski, S. Skowronski, A time delay system case study: computer integrated manufacturing and management system robustness. E. Petre (Ed.), $8^{\text {th }}$ IFAC Workshop on Time-Delay Systems, TDS'2009, Sinaia (Romania), 2009, Elsevier (Oxford, UK), p. 5.

[6] Jozef B. Lewoc, Antoni Izworski, Sławomir Skowronski, Antonina Kieleczawa, Peter Kopacek, An Integrated Manufacturing and Management System for Manufacturing Enterprises, JCIS, Vol. 1, No. 1, 2011, pp. 1-10,.

[7] J. B. Lewoc, A. Izworski, S. Skowronski, A. Kieleczawa, M. A. Hersh, P. Kopacek, Optimal Management Network Topology in a CIMM System: A Case Study IFAC Workshop on Supplemental Ways for International Stability, Waterford, 2012 (Submitted).

[8] Fall K. R., Stevens R. W., TCP/IP Illustrated Volume 1: The Protocols. Adison Wesley, 2011.

[9] Echelon, Introduction to LonWorks System. Available: www.echelon.com, 2012.

[10] F.P. Kelly, Reversibility and Stochastic Networks, Wiley, 1980.

[11] T. G. Robertazzi, Computer Networks and Systems. Queuing Theory and Performance Evaluation, Springer-Verlag, 2006.

[12] Robertazzi T., G., Networks and Grids. Springer, 2007.

[13] R. Serfozo, Introduction to Stochastic Networks, Springer-Verlag, 2006.

[14] J.B. Lewoc, Performance Evaluation Problems for Actual Computer Networks, AMSE Monographs, Tassin, 1990.

[15] J. B. Lewoc, A. Izworski, S. Skowronski, G. M. Dimirovski, V. Oljeska, Network Modelling Technique: A Case Study, IEEE (Eds.), Proceedings of CSNDSP (IEEE, IET), Newcastle, 2010.

[16] J. B. Lewoc, A. Izworski, S. Skowronski, A. Kieleczawa, T. Czachorski, G. M. Dimirovski, V. Oljeska, A Case Study: A Case Study: Performance Evaluation for a Computer Integrated Power Plant Network. Eurosim (Eds.) Proceedings of the 2010 Congress, Prague, Agresim, 2010 , p.6.

[17] J. B. Lewoc, A. Izworski, S. Skowronski, An Internal Traffic Modelling / Performance Measuring Tool. I. Troch, F. Breitenacker (Eds.), $5^{\text {th }}$ Vienna Symposium on Mathematical Modelling; $5^{\text {th }}$ MATHMOD, Vienna, Agrosim (Vienna), 2006, pp. 91-99.

[18] J. B. Lewoc, A. Izworski, S. Skowronski, Performance Modelling of a Computer Manufacturing and Management System. B. Zupancic, R. Karba, S. Blazic, Proceedings of the EUROSIM Congress on Modelling and Simulation, Ljubljana, Agresim (Vienna), 2008, p. 8. 